\section{Comment to the article by Arai Y, Jo T, Matsui H, \\ Kondo T and Takaori-Kondo A: "Comparison of up-front treatments for newly diagnosed immune thrombocytopenia - a systematic review and network meta-analysis". Haematologica 2018;103(1):163-171. Need to direct immune thrombocytopenia therapy towards shared goals}

On the basis of the results of their recent work titled "Comparison of up-front treatments for newly diagnosed immune thrombocytopenia - a systematic review and network meta-analysis", Arai and colleagues sustain that human thrombopoietin agonists may be beneficial up-front therapies in addition to conventional corticosteroid monotherapy in adults with primary immune thrombocytopenia, and that head-to-head trials including these regimens and rituximab-containing treatments are necessary to determine the most suitable therapies for these patients. Such head-to-head trials would, however, clash with the treatment currently foreseen for adults with newly diagnosed primary and severe immune thrombocytopenia which is not actually directed at modifying the natural history of the disease, but at 'achieving, rapidly, a safe platelet count to prevent or stop hemorrhages and to ensure an acceptable quality of life, avoiding, as much as possible, treatment-related adverse effects'. This fact and the lack of evidence that intensive medical therapy administered early in the disease course may improve or even cure immune thrombocytopenia mean that some treatments, including rituximab, are not, at present, acceptable as first-line therapy. In consideration of the potential usefulness of thrombopoietin agonists in up-front treatment for newly diagnosed immune thrombocytopenia, head-to-head trials similar to those proposed by Arai and colleagues would require a preliminary agreement on the goal of this line of therapy, just as we have sustained for other lines of immune thrombocytopenia therapy. This is to prevent exacerbating the disagreements already existing between different guidelines on the treatment of immune thrombocytopenia.

In a recent article in Haematologica, Arai and colleagues reported the results of a systematic review and network meta-analysis comparing the efficacy and safety of different treatments utilized in adults for primary newly diagnosed immune thrombocytopenia (ITP).

The study found that the main outcome, represented by a sustained response (platelet count $>30 \times 10^{9} / \mathrm{L}$ for $3-6$ months after completion of treatments), was achieved by a significantly higher proportion of patients treated with recombinant human thrombopoietin agonists + dexamethasone or rituximab + dexamethasone than by those treated with conventional prednisolone or dexamethasone monotherapy. Moreover, with regards to the two secondary endpoints considered, overall response (defined as a platelet count $>30 \times 10^{\circ} / \mathrm{L}$ for $2-4$ weeks after initiation of the upfront treatment) and therapy-related adverse events, thrombopoietin agonists + dexamethasone and thrombopoietin agonists + prednisolone improved early overall responses compared to prednisolone, dexamethasone, and rituximabcontaining regimens, while all treatments were tolerated and the therapy-related adverse event profiles were similar in all treatment arms. ${ }^{1}$

These results led the authors to claim that thrombopoietin agonists may be beneficial up-front therapies in addition to conventional corticosteroid monotherapies and that head-to-head trials including these regimens and rituximabcontaining treatments are necessary, in order to overcome the limitations of the small numbers in their study, to determine the most suitable therapies for newly diagnosed ITP. ${ }^{1}$

In our opinion, these conclusions did not take into consideration the need for a preliminary agreement on the goals that define the treatments foreseen for the different sequences of disease of primary ITP in adults, the so-called 'lines of therapy'. This is particularly true for second-line treatment, given the discordant suggestions for this line of therapy between the two most influential guidelines on the treatment of the disease, the 2010 International Consensus Report $^{2}$ and the 2011 guideline of the American Society of Hematology. ${ }^{3}$ Prior to conducting further trials it is of paramount importance to define shared treatment goals for the different lines of therapy for primary ITP in adults, as we have already underscored. ${ }^{4}$

Arai and colleagues stated the need for head-to-head randomized controlled trials in order to determine the most suitable initial treatment for newly diagnosed ITP in adults, but their conclusions were based on comparisons of various available treatments not considered by current guidelines. ${ }^{2,3}$ In fact, the goal of treatment foreseen for newly diagnosed adult patients with primary ITP requiring therapy ('first-line treatment') is not, at present, directed at modifying the natural history of the disease, ${ }^{5}$ but 'at achieving, rapidly, a safe platelet count to prevent or stop hemorrhages and to ensure an acceptable quality of life, avoiding, as much as possible, treatment-related adverse effects' ${ }^{6-8}$ This fact and the lack of evidence that intensive medical therapy administered early in the disease course may improve or even cure $\operatorname{ITP}^{9}$ mean that some treatments, including rituximab, are not, at present, acceptable as first-line therapy. ${ }^{6,10}$

\section{Lorenzo Cirasino and Stefano Semeraro \\ UO di Medicina, Ospedale di Ostuni, ASL BR, Italy \\ Correspondence: cirasino@telnetwork.it doi:10.3324/haematol.2018.189183}

Information on authorship, contributions, and financial \& other disclosures was provided by the authors and is available with the online version of this article at www. haematologica.org.

\section{References}

1. Arai Y, Jo T, Matsui H, Kondo T, Takaori-Kondo A. Comparison of up-front treatments for newly diagnosed immune thrombocytopenia - a systematic review and network meta-analysis. Haematologica 2018;103(1):163-171

2. Provan D, Stasi R, Newland AC, et al. International consensus report on the investigation and management of primary immune thrombocytopenia. Blood 2010;115(2):168-186.

3. Neunert C, Lim W, Crowther M, Cohen A, Solberg L Jr, Crowther MA. The American Society of Hematology 2011 evidence-based practice guideline for immune thrombocytopenia. Blood 2011;117(16):4190-4207.

4. Cirasino L, Semeraro S. Goals defining therapy for primary immune thrombocytopenia in adults. Blood Coagul Fibrinolysis 2017;28(4): 348-350.

5. Godeau B, Chevret S, Varet B, et al. Intravenous immunoglobulin or high-dose methylprednisolone, with or without oral prednisone, for adults with untreated severe autoimmune thrombocytopenic purpura: a randomized, multicentre trial. Lancet 2002;359(9300):23-29.

6. Rodeghiero F, Ruggeri M. ITP and international guidelines: what do we know, what do we need? Presse Med 2014;43(4 Pt 2):e61-e67.

7. Rodeghiero F, Stasi R, Germsheimer T, et al. Standardizations of terminology, definitions and outcome criteria in immune thrombocytopenic purpura of adults and children: report from an international working group. Blood 2009;113(11):2386-2393.

8. George JN. Definition, diagnosis and treatment of immune thrombocytopenic purpura. Haematologica 2009;94(6):759-762.

9. Cuker AD, Prak ET, Cines DB. Can immune thrombocytopenia be cured with medical therapy? Semin Thromb Hemost 2015;41(4):395404.

10. Chug S, Darvish-Kazem S, Lim W, et al. Rituximab plus standard of care for treatment of primary immune thrombocytopenia: a systematic review and meta-analysis. Lancet Haematol 2015; 2(2):e75-e81. 